\title{
OPEN SOURCE CLOUD-BASED TECHNOLOGIES FOR BIM
}

\author{
Sotirios Logothetis, Eleni Karachaliou, Elena Valari, Efstratios Stylianidis \\ Faculty of Engineering, School of Spatial Planning \& Development \\ Aristotle University, Thessaloniki, 54124, Greece \\ slogothet@auth.gr, ekaracha@auth.gr, evalarig@auth.gr, sstyl@auth.gr
}

\author{
Commission II, WGII/2
}

\begin{abstract}
KEY WORDS: Building Information Modelling (BIM), Heritage BIM (HBIM), Cloud computing, Open Source Software, BIMserver,
\end{abstract} Nextcloud, FreeCAD, collaboration

\begin{abstract}
:
This paper presents a Cloud-based open source system for storing and processing data from a 3D survey approach. More specifically, we provide an online service for viewing, storing and analysing BIM. Cloud technologies were used to develop a web interface as a BIM data centre, which can handle large BIM data using a server. The server can be accessed by many users through various electronic devices anytime and anywhere so they can view online 3D models using browsers. Nowadays, the Cloud computing is engaged progressively in facilitating BIM-based collaboration between the multiple stakeholders and disciplinary groups for complicated Architectural, Engineering and Construction (AEC) projects. Besides, the development of Open Source Software (OSS) has been rapidly growing and their use tends to be united. Although BIM and Cloud technologies are extensively known and used, there is a lack of integrated open source Cloud-based platforms able to support all stages of BIM processes. The present research aims to create an open source Cloud-based BIM system that is able to handle geospatial data. In this effort, only open source tools will be used; from the starting point of creating the 3D model with FreeCAD to its online presentation through BIMserver. Python plug-ins will be developed to link the two software which will be distributed and freely available to a large community of professional for their use. The research work will be completed by benchmarking four Cloud-based BIM systems: Autodesk BIM 360, BIMserver, Graphisoft BIMcloud and Onuma System, which present remarkable results.
\end{abstract}

\section{INTRODUCTION}

Building Information Modelling (BIM) is defined as the method by which the design, implementation and management of buildings and other structures are integrated (Matthews et al., 2015). The US National BIM Standards Committee definition (NBIMS, 2018) of BIM concerns "an integrated digital representation of the physical and functional characteristics of an infrastructure".

The last decade, a remarkable development and use of BIM technology in the field of cultural heritage has been occurred and so the term HBIM was introduced. Cultural heritage elements are gathered using a terrestrial laser scanner, and produce photogrammetric survey data. Therefore, the data transformed into parametric objects with unique standards and protocols that can be exchanged and processed from different experts (Osello and Rinaudo, 2016) in order to document any size or complex form of cultural heritage (Singh et al., 2011).

Cloud computing services adoption in the BIM and HBIM field is appealing. The Cloud services refers to a network that is used to interconnect with multiple computers or to get services provided by a remote host over a network connection. Network access is acceptable in a shared set of customisable computing resources (such as networks, servers, storage, applications, and services). Compared with the usual personal information systems, Cloud computing has powerful computer competences. Furthermore, it can handle a large amount of data and provide various types of software services. Cloud technology offers remarkable savings in companies' resources by using cheaper computing power. Therefore, it helps at their technological progression (Vaquero et al., 2009).

Most professionals mainly use a stand-alone commercial platform for BIM projects. In fact, recent research (Logothetis \& Stylianidis, 2016) revealed that except of a few, accessible and free BIM viewers there is no available, a valuable and comprehensive free or open source BIM software to be used for the overall BIM process. Chen et al. (2016) underpin that the most significant drawbacks of using a standalone platform for BIM applications are as follows:

1. The computer is often overburdened by using BIM software due to constrained resources.

2. It has problem managing multiple BIM projects.

3. It is a hostile data-sharing environment for multiple users collaborating on the same project.

4. It has difficulty answering the needs of statistical analysis on a BIM project.

5. It has difficulty to store and share large data files.

6. The price of the commercial BIM software are very high and inaccessible. 
Responding to these issues, this study presents an open source BIM platform developed using Cloud computing technology that is able to handle geospatial data. The software that used for BIM or HBIM processes are the same. For this reason, we will generally refer to the BIM term for software, applications, projects etc. as it includes both categories.

\section{RELATED RESEARCH REVIEW}

In this paper, we start with the literature review, illuminating the published research in the field of BIM, Cloud computing, and their integration. The following papers are those contributed significantly in this study.

Chong et al. (2014) mentioned that fragmentation of practices is one of the key issues in BIM. However, with advances particularly in Cloud computing and in Information and Communication Technology (ICT), the fragmentation of working practices can be probably overcome. The ICT could enhanced information flowing and communication in various stages along a project life cycle.
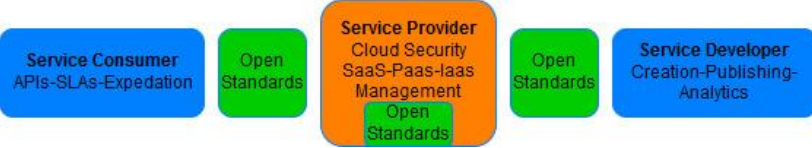

Figure 1. Taxonomy of Cloud computing (Chong et al., 2014)

Chen et al. (2016) presented a Cloud-based system framework based on MapReduce and Bigtable as the data storage and processing paradigms for providing a web service for storing, viewing, and analysing massive Building Information Models (BIMs). Cloud, WebGL and Web 3D technologies were utilized to develop a BIM data centre that can be accessed by users to submit and view BIMs online in 3D.

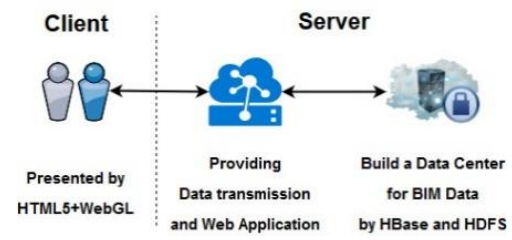

Figure 2. System for data storage and $3 \mathrm{D}$ viewing of massive BIMs (Chen et al., 2016)

Polter and Scherer (2017) introduced the BIMgrid, which is an adaptive infrastructure integration framework for civil engineering applications, based totally on public Cloud resources and private grid. The BIMgrid is designed as a layered serviceoriented architecture (SOA) and offers several interfaces. The data management supports the interoperability between applications and will be based on the Industry Foundation Classes (IFC) standard and vendor specific data formats.

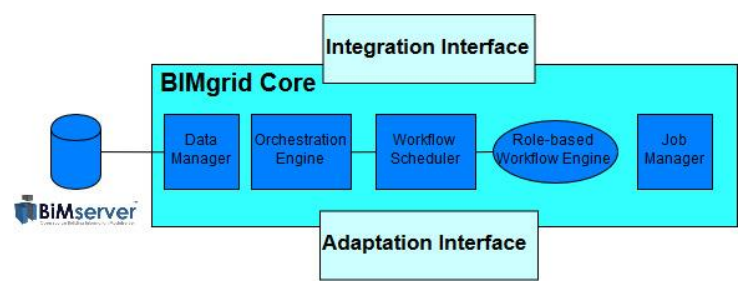

Figure 3. BIMgrid interface architecture (Polter and Scherer, 2017)
Du et al. (2018) mentioned Virtual Reality (VR) shows a great potential to improve BIM workflow efficiency through enhanced common understanding. However, with VR there is a lack of automated and efficient data transfer approach between BIM. They suggested a BIM-VR (BVRS) real-time synchronisation system, which is grounded on a Cloud-based BIM metadata communication and interpretation method. In several tests the BVRS allowed users to update BIM model changes simultaneously and automatically. The results of using the BVRS have confirmed their usability and efficiency.

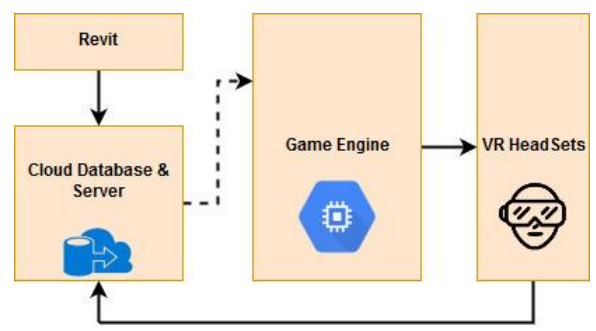

Figure 4. Architecture of BVRS (Du et al., 2018)

\section{CLOUD BIM PROGRAMS}

Cloud technology is very well known as an alternative and cost operative answer to current state of data storage and exchange. Since potential values of programs based in Cloud technology such as low-cost and efficiency has been recognised, is believed to be a promising trend the combination of BIM and Cloud computing. Recently, there is tendency in the BIM programs to gradually integrated and supported Cloud services. As a result, Cloud-based BIM programs and BIM web services are becoming more and more popular (Afsari et al., 2016). The integrated Cloud-BIM model considered to be the second generation of BIM programs. It enables a more effective real-time communication platform and higher levels of collaboration for project team members. However, the Cloud technology is still relatively new to the construction industry (Li et al., 2014).

Redmond et al. (2012) carried out interviews in 11 experts to discover how the use of Cloud BIM affects positively the information exchange process. They concluded that it is reasonable to have opportunities for different disciplines to exchange and share the necessary data for making key decisions at the early design stage. Porwal and Hewage (2013) had suggested that the integration of Cloud computing with BIM partnering framework could facilitate the pre-planning of sustainable construction throughout a project's lifecycle.

The services of the Cloud can be deployed as Public, Private, Community and Hybrid (Kumar et al., 2018). According to Bilal et al. (2016) and NIST (Web 1), as shown in the Figure 5, Cloud computing depending on the management of services can be split into three main services SaaS, PaaS and IaaS (SPI model):

1. Software-as-a-service (SaaS): a user via the Internet has access to enterprise applications.

2. Platform-as-a-service (PaaS): a user is provided with services pertaining to development environments such as programming languages, operating systems or databases, etc.

3. Infrastructure-as-a-service (IaaS): a user is provided with computer and network infrastructures as a fully outsourced service. 


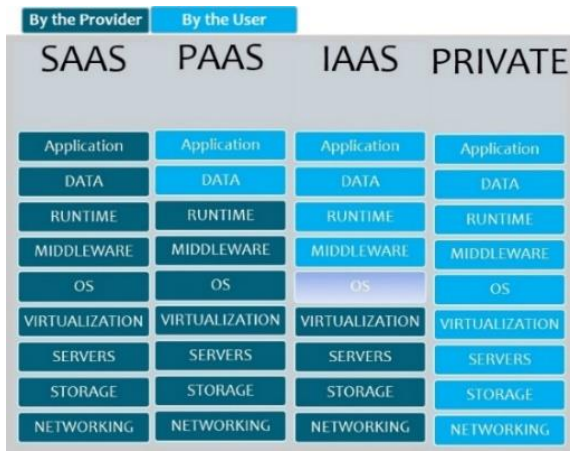

Figure 5. Security Responsibility in the Cloud (Web 17)

For a private Cloud, the administrator of the organization has more flexibility to customise how servers, networks, and storage are separated.

In this paper we compared a total of four Cloud-based BIM programs, namely Autodesk BIM 360, BIMServer, Graphisoft BIMcloud and Onuma System. All programs fulfil the definition of a BIM, which enables digital representation of the physical and functional characteristics of a structure and also geospatial data. The results presented in Table 1, and can be further used in order to draw useful conclusions. It can be clearly perceived that Autodesk BIM360 and Graphisoft BIMcloud offers the most of the features, while BIMserver provides the fewest ones. On the other side, BIMserver is the only program labelled as Free and Open Source Software (FOSS).

\begin{tabular}{|l|c|c|c|c|}
\hline Software Features & \multicolumn{3}{|c|}{ Cloud-based BIM programs } \\
\hline & BIM & BIM & BIM & Onuma \\
& 360 & server & cloud & System \\
Design tools & $\checkmark$ & & $\checkmark$ & $\checkmark$ \\
3D visualization & $\checkmark$ & $\checkmark$ & $\checkmark$ & $\checkmark$ \\
Online file storage & $\checkmark$ & $\checkmark$ & $\checkmark$ & $\checkmark$ \\
Online drawing viewing & $\checkmark$ & & $\checkmark$ & $\checkmark$ \\
Online drawing editing & $\checkmark$ & & $\checkmark$ & $\checkmark$ \\
Clash detection & $\checkmark$ & $\checkmark$ & & \\
Lifecycle Management & $\checkmark$ & & & $\checkmark$ \\
Simulations & $\checkmark$ & $\checkmark$ & $\checkmark$ & $\checkmark$ \\
Detailed management & $\checkmark$ & & $\checkmark$ & \\
permissions & & & & \\
Online sharing of file & $\checkmark$ & $\checkmark$ & $\checkmark$ & $\checkmark$ \\
Mobile apps & $\checkmark$ & & $\checkmark$ & $\checkmark$ \\
Browser based & $\checkmark$ & $\checkmark$ & $\checkmark$ & $\checkmark$ \\
management & & & & \\
Folder hierarchy & $\checkmark$ & $\checkmark$ & $\checkmark$ & $\checkmark$ \\
Support IFC files & $\checkmark$ & $\checkmark$ & $\checkmark$ & $\checkmark$ \\
File management system & $\checkmark$ & $\checkmark$ & $\checkmark$ & $\checkmark$ \\
Clash detection & $\checkmark$ & $\checkmark$ & $\checkmark$ & \\
Private Cloud & & $\checkmark$ & $\checkmark$ & $\checkmark$ \\
Encrypted data & $\checkmark$ & & $\checkmark$ & $\checkmark$ \\
http/https support & $\checkmark$ & $\checkmark$ & $\checkmark$ & $\checkmark$ \\
Multiple Cloud Servers & & & $\checkmark$ & \\
Automated server backups & & & $\checkmark$ & \\
User groups & $\checkmark$ & & $\checkmark$ & \\
Multi language support & $\checkmark$ & & $\checkmark$ & \\
Free trial & $\checkmark$ & $\checkmark$ & & \\
Free or Open Source & & $\checkmark$ & & \\
\hline
\end{tabular}

Table 1. Comparison of features of Cloud-based BIM programs ((Chong et al., 2014) \& Web 2, 3, 4, 7)
Autodesk BIM360 is especially a design software with all the planning functions. The additional Cloud computing capabilities, are provided as more options. The software can be either established in the computer or used thru the Cloud, providing users the best of both capabilities (Chong et al., 2014)

BIMServer enables users to create a BIM Cloud of their own. This Cloud can be either through a Cloud service provider or a private Cloud. Primarily, this software is a file and BIM sharing program which allows the users collaborate in a project (Chong et al., 2014).

Another Cloud-based BIM software is BIMcloud, which allows a BIM workflow on any hardware, software or network configuration, connecting users, teams and projects of any size or setup. It combines "Delta" BIM Server technology with the advantages of the Cloud computing (Web4).

Finally, Onuma System has varied functions in its Cloud computing options too. The strength of this software is towards architectural use and the project management aspect. In addition to that, it works well for project and program development, while data from other users can exported or imported in this software as well (Chong et al., 2014).

\section{BIM DATA}

BIMs used in the AEC industry usually contain 3D information that primarily refers to vector-based data. On the other hand, BIMs are also used for the representation and management of existing structures, commonly described with the term "as-built" BIM. The development of an "as-built" BIM requires the data acquisition of the current state of the relevant structure (Macher et al., 2017), the geometrical modelling of the objects, the attribution of categories and material properties to the objects and the creation of relations between them (Hichri et al., 2013). In this case, the use of 3D point cloud data of the built environment, providing the real capture of the field conditions, serves as a solution to enhance 3D BIM applications (Qu and Sun, 2015).

The term "point cloud" refers to a set of points in a coordinate system usually representing the surface of an object or area. When referring to a three-dimensional coordinate system these points are defined by $\mathrm{x}, \mathrm{y}$ and $\mathrm{z}$ spatial coordinates (Qu and Sun, 2015). The most common used techniques to acquire 3D point cloud data are range-based i.e. lasergrammetry, or image-based i.e. photogrammetry ( $\mathrm{Qu}$ and Sun, 2015) \& (Hichri et al., 2013). Lasergrammetry refers to the use of different kinds of laser scanners (i.e. long-range scanners, terrestrial laser scanners, triangulation scanners etc.), in order to collect high-density point cloud data by projecting a laser beam on the geometry to be captured. Photogrammetry is a technique to create 3D representations using overlapping images of the interested object/area (Hichri et al., 2013).

Qu and Sun (2015) indicate that in the construction project lifecycle using BIM, 3D point cloud data may be used during:

- The planning and design phase, in order to acquire data of the surrounding area in a quick and low-cost way.

- The bidding and construction phase providing the constructor the opportunity to extract dimensional information and make realistic decisions on the work progress. 
- The post-construction phase to update the construction BIM models or to compare before and after.

Although the integration of 3D point clouds in BIM offers many advantages and facilitates the design process, however, two major challenges remain ((Qu and Sun, 2015), (Macher et al., 2017), (Hichri et al., 2013)):

- In comparison to the vector-based data, the point cloud contains raster geometric information and therefore the amount of data is huge which means high requirements in terms of computer storage and computation load.

- The integration of a 3D point cloud into BIM is considered time-consuming, since the point cloud contains only geometric information and the characterisation of object shapes, relations and attributes needs to be completed manually. Especially, when it comes to complex geometries, such as cultural heritage structures that are characterized by various uncategorized shapes.

In a BIM workflow, data are managed and shared between AEC professionals, users, and systems. The file formats are commonly used are proprietary (like: RVT, OBJ, STL, DWF, DXF, etc.) and non-proprietary (like: IFC) for data sharing between BIM software packages (Bilal et al., 2016).

IFC by buildingSMART, formerly the International Alliance for Interoperability (IAI), is an open and neutral data format that is compatible with various BIM applications. It is the only public standard for BIM data exchanges that includes geometry, object structure (topology), material and performance attributes (Kim et al., 2015). The IFC is used for sharing of information between the project, design, maintenance, procurement, and operations teams (Web 5).

\section{THE PROPOSED CLOUD-BASED SYSTEM}

The proposed Cloud-based system for providing a web-based service for managing BIM data is called CloudServerBIM. It is a real-time web service provided at the same time to multiple users for viewing and uploading BIM data anywhere, anytime, using any computer or mobile device. It makes use only FOSS, such as Ubuntu ver.16.04 LTS (Web 6) as the operating system, BIMserver ver.1.5.95 (Web 7) as the BIM data file server, and Nextcloud ver.13 (Web 8) as a file share and communication platform.

Ubuntu is a Linux distribution based on the Debian architecture. It is an open source operating system for computers. Ubuntu is also available for smartphones and tablets, with the so-called Touch edition (Web 9). It is popular on network servers with enterprise-class features, usually running the Server variant (Web $6)$.

Nextcloud, as shown in Figure 6, is an open source client-server program for developing and using file hosting services. It is functionally similar to Dropbox, permitting anyone to install and operate it on a personal server. Some of the key Nextcloud's features are:

1. User files are encrypted.

2. Data can be shared by defining read/write permissions between users or groups.

3. It permits user and group administration via LDAP or OpenID.

4. Users can create public URLs when sharing files (Web $8)$.

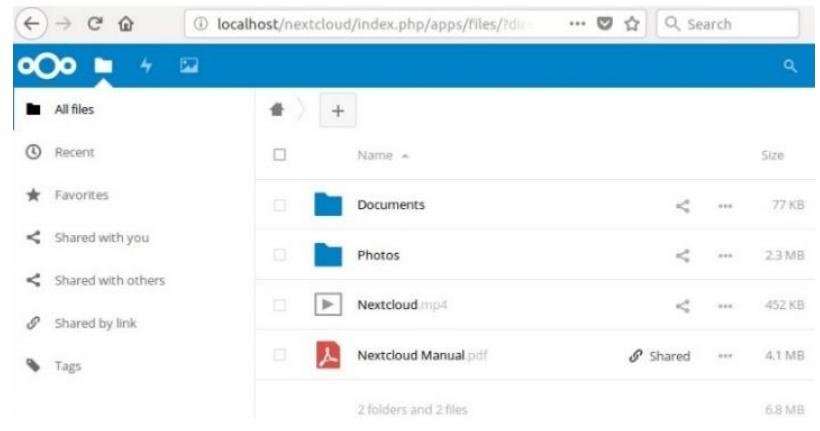

Figure 6. File - directory structure of the Cloud

BIMServer, as illustrated in Figure 7, is a FOSS that enables users to transform any computer into a file sharing BIM server. It has the ability to analyse IFC data and files. IFC data are interpreted and stored as objects in a specific database. On top of the database core features like model checking, comparing, merging, authorisation/authentication, etc. are available. The files located in the server will be available via a web browser. BIMServer is used in the AEC industry for effective and efficient collaboration (Chong et al., 2014).

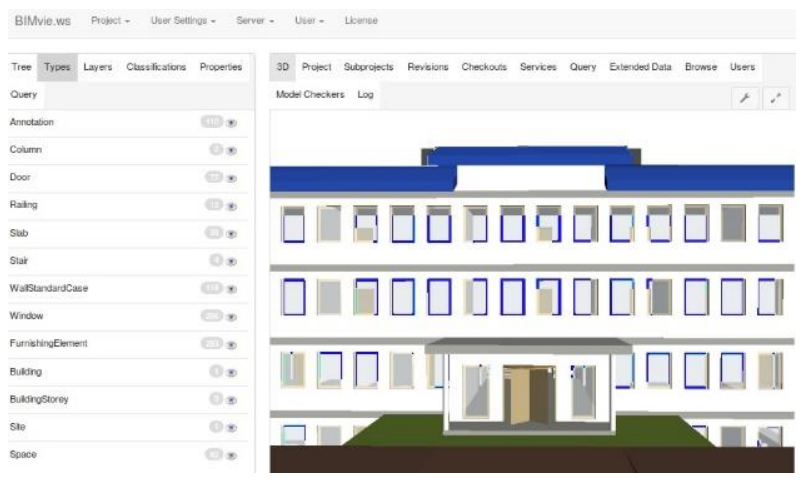

Figure 7. BIMserver: IFC on BIMvie.ws

The CloudServerBIM belongs to the category of a private Cloud. A private Cloud is created and managed by an organisation, providing much more privacy and control (Mahamadu et al., 2013). According to Liu et al. (2016) the characteristics of a private Cloud are:

1. Scalable: high levels of utilisation (through virtualisation of the data centres).

2. Shared: capacity is pooled as the workloads are multiplexed.

3. Accessible: easy to use the Cloud-based web services. IT users can self-provision.

4. Elastic: ability to dynamically add or reduce resource according to the user's requirements (e.g. infinite capacity on demand, integrate with public Cloud, etc.).

5. Cost-effective: minimising the budget of the organisation.

\subsection{System requirements}

There are three fundamental requirements for realising an online web system for multi-user BIM data collaboration and managing. First, with regard to the model presentation, the system should display online all the components in a $3 \mathrm{D}$ presentation. Then, it should permit users to access the properties of the BIM 
components. Second, data security is very important in terms of user cooperation through the Cloud system. Third, as far as the performance of the system is concerned, the Ubuntu operating system should be reliable, stable, extremely fast, and without requiring high computing resources.

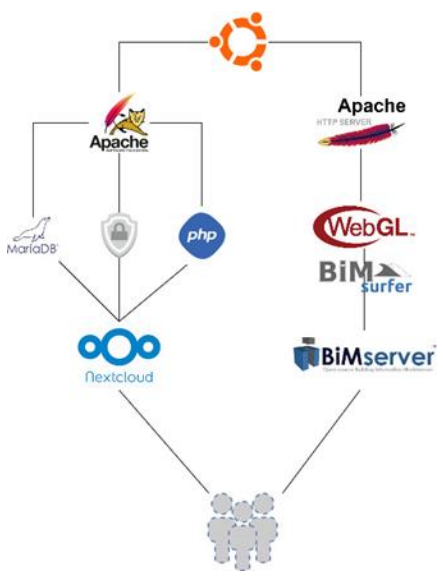

Figure 8. CloudServerBIM architecture

In this study, the following technologies integrated to develop the CloudServerBIM, which satisfy these requirements. CloudServerBIM architecture illustrated in Figure 8, while the embedded technologies described below.

1. Apache HTTP Server, is a free and open-source web server. It is a cooperative development aimed at creating a robust, and freely available source code implementation of a Web server. The Apache project is managed by a group of volunteers in the world, using the Internet. Apache supports a variety of programming languages such as PHP, Python, Tcl and Perl. It also includes popular authentication modules, Secure Sockets Layer, Transport Layer Security, a proxy module, a URL rewriting module, custom log files and filtering. It supports digital certificate authentication and password authentication. Apache features DBMS-based authentication databases, content negotiation, and configurable error messages. Several graphical user interfaces (GUIs) supported (Web 11).

2. The Apache Tomcat is an open source implementation of the Java Servlet Container developed by the Apache Software Foundation. Tomcat provides an HTTP web server in which Java code can run. It is developed and maintained under the auspices of the Apache Software Foundation, by an open community of developers. In this project, Tomcat 8.0.49 used, which implements the JSP (Java Server Pages) 2.4 and Servlet 3.1 Specifications (a Java program that extends the capabilities of a server) (Web 12). This is a necessary tool for BIMserver operation.

3. The Cloud services are provided by the Nextcloud client-server software. Prerequisites for proper operation of the Nextcloud are the pre-installation of the Apache2 HTTP server, a MySQL and MariaDB database (a database server like MySQL (Web 13)), the PHP 7.1 (a server scripting language (Web 14)) and SSL/TLS cryptographic protocols (Secure Sockets Layer/Transport Layer Security) for data encryption which is also necessary for data security (Polter and Scherer, 2017).

4. The BIMserver provides a suitable solution for IFC based data management (storing, maintaining, and querying IFC files). It provides the "BIM Surfer", an open source web-based (WebGL extension) viewer toolkit for the visualisation of BIM data from IFC (Web 15). It also provides the BIMviews software a tool to evaluate and view BIM models online (Web 16). BIMServer architecture is presented in Figure 9. It centralises the information of a project with a core that is based totally on IFC data. Finally, it shares this information to client applications through specialised interfaces ("BIM Surfer" and BIMviews).

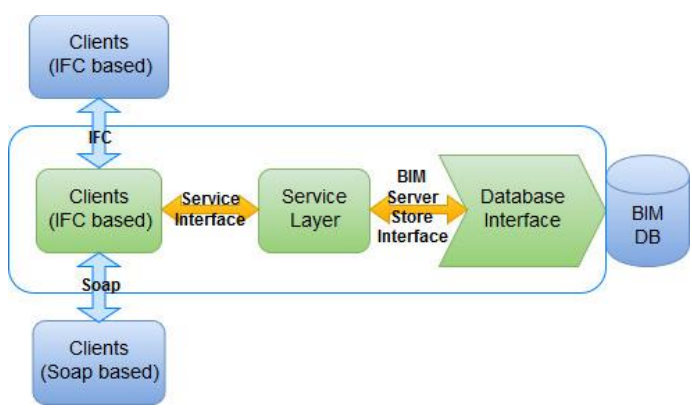

Figure 9. BIMServer architecture (Afsari et al., 2016)

\subsection{System architecture}

Figure 10 shows the architecture of the proposed CloudServerBIM collaboration system for data storage and 3D BIM data viewing. The system is divided into two layers: the back-end and the front-end. Front-end is all the structure involved and what a user is interfacing, which includes some programming languages like PHP, Javascript and HTML. Back-end refers to scripts or programs that are executed on the server itself and are independent from the client's PC.

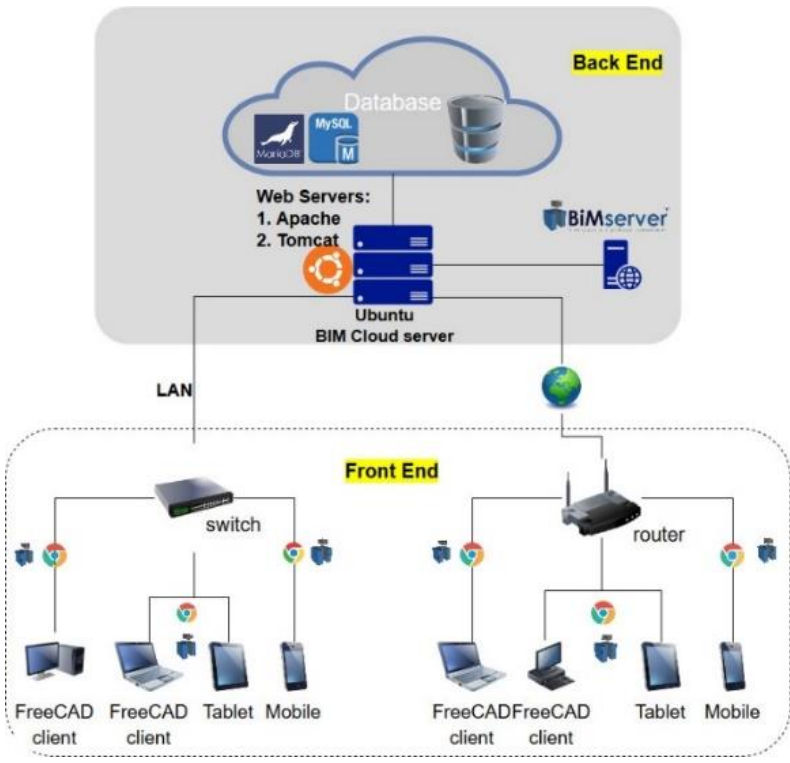

Figure 10. The proposed CloudServerBIM

In detail, back-end is the private server with Ubuntu operating system. The following programs are installed on it: Nextcloud, BIMserver, Apache2 HTTP server, Apache Tomcat8. The database systems that's used is MariaDB installed alongside MySQL. Users can access the server as well as the services it offers (Cloud \& BIMserver) either through a local network, or over the Internet.

At the front-end layer, users can create BIM models using FreeCAD software. FreeCAD is a FOSS parametric 3D CAD modeller (Web 10) that is also used for BIM modelling 
(Logothetis et al., 2018). Users use the FreeCAD that is installed on their computer for BIM modelling. They can store their data either on the local disk or on Cloud via the local network or over the Internet. BIM data in the Cloud, can be shared with other project participants. BIMserver enables users to view BIM models and IFC files using their personal computers or mobile devices. The benefits of using this proposed Cloud system are:

- $\quad$ large storage space;

- effective teamwork:

- $\quad$ safety in storing data in the Cloud system;

- $\quad$ easy public access to data stored in the Cloud system; and

- Web-based accessibility (web-interface).

In CloudServerBIM as shown in Figure 11, users can be divided into two types: BIM-viewers and BIM-uploaders. The two types of users are described in detail below:

1. BIM-viewers connect to the server and select the BIM data they want to view. BIMserver with the "BIM Surfer WebGL viewer" retrieves the BIM geometry data from private Cloud and presents it to users. In the illustrated BIM, users can change viewing angles, adjust the view, and select the objects to view their properties.

2. BIM-uploaders connect to the server using various digital devices (computers, tablets, and smartphones). They transfer IFC files to the Cloud system, which are then analysed and converted into 3D formats. These formats can be read by "BIM Surfer" and WebGL on the client-side. Finally, the properties of BIM data are extracted and stored in the private Cloud system.

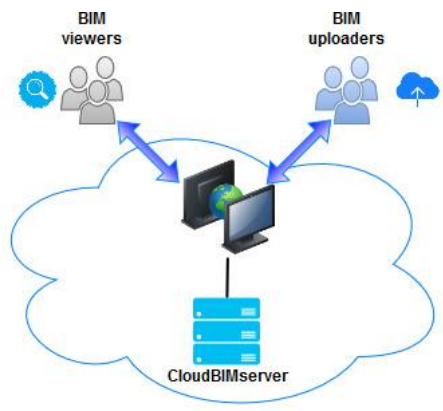

Figure 11. System framework with two types of users (viewers and uploaders)

The files shared or stored through the CloudServerBIM come either from Photogrammetry processes, 3D scanning or from data processing with a BIM software.

\subsection{System validation}

Validation of the CloudServerBIM was conducted on one computer with Intel Core i3 CPU, 8GB RAM, 1TB hard disk, and Linux Ubuntu 16.04 LTS as operating system. Ubuntu is free and was built on a world of the open source projects (Ho et al., 2018). The computer served as login server, equipped with BIMserver and Nextcloud. BIMserver is equipped with two plugins, the "BIM Surfer" (an open source WebGL based IFC uploaderviewer) and the BIMviews (a Javascript plugin for uploadingviewing 3D BIM models). This computer, in combination with the above programs, formed a small private Cloud.
Two other computers with similar hardware specifications and FreeCAD installed, were used for BIM modelling (Logothetis et al., 2018). These computers were used to upload and view BIM models. A smartphone and a tablet, were also used only for viewing these models via BIMserver. It is important to point out that many users can simultaneously view BIM models online using the CloudServerBIM system. The models are displayed in a browser (e.g. Chrome, Firefox, etc.) via the local network or the Internet. The BIMserver and Nextcloud web address is available on the network through the Apache HTTP and Tomcat Server.

Performance in the presentation of BIM models depends on the speed of the network and the specifications of users' display devices. When users start viewing the model (e.g. IFC), the system retrieves from the server all the components of the BIM model. In addition, the system supports simultaneous uploading of BIM data by multiple users. The storage space of the Cloud system, that is available to users for file sharing and teamwork, depends on the capacity of the server's hard disk.

\section{CONCLUSIONS}

Existing Cloud-based applications for BIM face several challenges in data exchange and have not fully exploit the potential of the Cloud. Therefore, there is a need to develop a new free and open source system that defines the collaborative processes and BIM file sharing utilising network technologies as major Cloud enablers.

This study proposed an open source Cloud-based system framework for storing, viewing, and analysing BIM and HBIM models. For the development of the system, we combined different technologies; Cloud computing technology with the Nextcloud software, 3D display technology with BIMserver equipped by plugins "BIM Surfer" and BIMviews, and PHP web technology. The proposed system framework can provide web services that allow AEC professionals to upload BIM and HBIM models to the Cloud data centre for sharing them with others involved in a project. The system, which might viewed as a BIM and HBIM reviewing platform that facilitates the collaboration, is divided into a client-side and a server-side.

The client-side contains the FreeCAD as the modelling software and a browser, connected with the server-side that provides PHP and WebGL 3D technologies. The user can use any computer or mobile device to connect to the network and then connect into CloudServerBIM anytime and from anywhere, to view or upload BIM and HBIM models. The server-side includes Ubuntu operating system, the BIMserver and Nextcloud. BIMserver enables to create an online BIM system, which handles IFC data. In close collaboration with the Nextcloud, it supports dynamic teamwork process in the AEC sector.

This study has also identified and reviewed four Cloud-based applications that are suitable for BIM. Autodesk BIM 360, BIMServer, Graphisoft BIMcloud, and Onuma System were analysed properly. Graphisoft BIMcloud and Autodesk BIM360 offer most of the features, while BIMserver provides the fewest features. However, BIMserver is source FOSS. The program core is based on the open standard IFC and for that reason is a perfect choice for those users who have limited financial resources for IFC data management. Many software engineers and many AEC applications use the BIMserver platform as the reliable stable base, and build upon their own tools. Putting BIMserver and 
Nextcloud work together, is the ideal solution to creating a Cloud-based system for BIM and HBIM.

Our future research agenda will focus on improving the CloudServerBIM efficiency and data security. The application of the proposed Cloud-based system for specialised BIM and HBIM is also an underexplored area of our research.

\section{REFERENCES}

Afsari, K., Eastman, C.M., Shelden, D.R., 2016. Cloud-based BIM Data Transmission: Current Status and Challenges. 33rd International Symposium on Automation and Robotics in Construction. https://doi.org/10.22260/ISARC2016/0129

Bilal, M., Oyedele, L.O., Qadir, J., Munir, K., Ajayi, S.O., Akinade, O.O., Owolabi, H.A., Alaka, H.A., Pasha, M., 2016. Big Data in the construction industry: A review of present status, opportunities, and future trends. Advanced Engineering Informatics 30, pp. 500-521. doi:10.1016/j.aei.2016.07.001

Chen, H., Chang, K., Lin, T., 2016. A cloud-based system framework for performing online viewing, storage, and analysis on big data of massive BIMs. Automation in Construction 71. doi:10.1016/j.autcon.2016.03.002

Chong, H., Wong, J.S., Wang, X., 2014. An explanatory case study on cloud computing applications in the built environment. Automation in Construction 44, pp. 152-162. doi:10.1016/j.autcon.2014.04.010

Du, J., Zou, Z., Shi, Y., Zhao, D., 2018. Zero latency: Real-time synchronization of BIM data in virtual reality for collaborative decision-making. Automation in Construction 85, pp. 51-64. doi:10.1016/j.autcon.2017.10.009

Hichri, N., Stefani, C., Luca, L.D.E., Veron, P., Hamon, G., 2013. From point cloud to BIM: a survey of existing approaches. XXIV International CIPA Symposium, France, 2013 - Proceedings of the XXIV International CIPA Symposium.

Ho, S.M., Kao, D., Wu, W.-Y., 2018. Following the breadcrumbs: Timestamp pattern identification for cloud forensics. Digital Investigation 24, pp. 79-94. doi:10.1016/j.diin.2017.12.001

Kim, M.K., Cheng, J.C.P., Sohn, H., Chang, C.C., 2014. A framework for dimensional and surface quality assessment of precast concrete elements using BIM and 3D laser scanning. Automation in Construction 49, pp. 225-238. doi:10.1016/j.autcon.2014.07.010

Kumar, P.R., Raj, P.H., Jelciana, P., 2018. Exploring Data Security Issues and Solutions in Cloud Computing. Procedia Computer Science 125, pp.691-697. doi:10.1016/j.procs.2017.12.089

Li, H., Wong, J., Wang, X., Li, H., Chan, G., 2014. A review of cloud-based BIM technology in the construction sector. Journal of Information Technology in Construction, vol.19, pp. 281-291.

Liu, X.L., Sheu, R.K., Yuan, S.M., Wang, Y.N., 2016. A filededuplicated private cloud storage service with CDMI standard. Computer Standards \& Interfaces 44, pp. 18-27. doi:10.1016/j.csi.2015.09.010
Logothetis, S. and Stylianidis, E., 2016. BIM Open Source Software (OSS) for the documentation of Cultural Heritage. Proc. 8th International Congress on Archaeology, Computer Graphics, Cultural Heritage and Innovation 'ARQUEOLÓGICA 2.0', pp. 28-35.

Logothetis, S., Valari, E., Karachaliou, E., Stylianidis, E., 2018. Development of an Open Source Spatial DBMS for a FOSS BIM. In Latest Developments in Reality-Based 3D Surveying and Modelling. MDPI: Basel, Switzerland, pp. 326-347.

Macher, H., Landes, T., Grussenmeyer, P., 2017. From Point Clouds to Building Information Models: 3D Semi-Automatic Reconstruction of Indoors of Existing Buildings. Appl. Sci. 7, 1030. doi:10.3390/app7101030

Mahamadu, A.M., Mahdjoubi, L., Booth, C., 2013. Challenges to BIM-cloud integration: Implication of security issues on secure collaboration. Proc. International Conference on Cloud Computing Technology and Science, pp. 209-214. doi:10.1109/CloudCom.2013.127

Matthews, J., Love, P.E.D., Heinemann, S., Chandler, R., Rumsey, C., Olatunj, O., 2015. Real time progress management: Re-engineering processes for cloud-based BIM in construction. Automation in Construction 58, pp. 38-47. doi:10.1016/j.autcon.2015.07.004

Osello, A. and Rinaudo, F., 2016. Cultural Heritage Management Tools: The Role of GIS and BIM. 3D Recording, Documentation and Management of Cultural Heritage. Dunbeath: Whittles Publishing, pp.105-124.

Polter, M., Scherer, R., 2017. Towards an adaptive civil engineering computation framework. Procedia Engineering 196, Creative Construction Conference, pp. 45-51. doi:10.1016/j.proeng.2017.07.171

Qu, T. and Sun W., 2015. Usage of 3D Point Cloud Data in BIM (Building Information Modelling): Current Applications and Challenges. Journal of Civil Engineering and Architecture 9, pp. 1269-1278. doi:10.17265/1934-7359/2015.11.001

Redmond, A., Hore, A., Alshawi, M., West, R., 2012. Exploring how information exchanges can be enhanced through Cloud BIM. Automation in Construction 24, pp. 175-183. doi:10.1016/j.autcon.2012.02.003

Singh, V., Gu, N., Wang, X., 2011. A theoretical framework of a BIM-based multi-disciplinary collaboration platform. Automation in Construction 20(2), pp. 134-144. https://doi.org/10.1016/j.autcon.2010.09.011

US National BIM Standards Committee (NBIMS). https://www.nationalbimstandard.org (14 March 2018)

Vaquero, L.M., Rodero-Merin, L., Cáceres, J., Lindner, M., 2009. A break in the clouds: towards a cloud definition. ACM SIGCOMM Computer Communication Review 39(1), pp.50-55. doi: 10.1145/1496091.1496100

Web 1, National Institute for Standards and Technology (NIST) Special Publication 500-299, https://bigdatawg.nist.gov/_uploadfiles/M0007_v1_3376532289 .pdf (20 March 2018) 
Web 2, Autodesk BIM 360, Construction management software for improved decision-making, https://bim360.autodesk.com (12 March 2018)

Web 3, Onuma, http://www.onuma-bim.com (12 March 2018)

Web 4, Overview - Graphisoft BIMcloud, http://www.graphisoft.com/bimcloud/overview (12 March 2018)

Web 5, buildingSMART, https://www.buildingsmart.org (19 March 2018)

Web 6, Ubuntu, https://www.ubuntu.com (3 March 2018)

Web 7, Open Source BIMserver, http://bimserver.org (12 March 2018)

Web 8, Nextcloud, https://nextcloud.com (18 March 2018)

Web 9, Ubuntu Touch, https://ubports.com (29 March 2018)

Web 10, FreeCAD: An open-source parametric 3D CAD modeler, https://www.freecadweb.org (1 April 2018)

Web 11, The Apache HTTP Server Project, https://httpd.apache.org (4 March 2018)

Web 12, The Apache Tomcat Project, http://tomcat.apache.org (4 March 2018)

Web 13, The MariaDB project, https://mariadb.org (7 March 2018)

Web 14, PHP, http://php.net/ (8 March 2018)

Web 15, Open source WebGL viewer for IFC models, http://bimsurfer.org/ (16 March 2018)

Web 16, The BIMviews software, http://bimvie.ws (26 March 2018)

Web 17, Cloud Security Part 1: Planning, Governance, MultiTenancy, http://crmtrilogix.com/Cloud-Blog/Security/CloudSecurity-Part-:--Planning-Governance-Multi-Tenancy/233 (28 March 2018) 